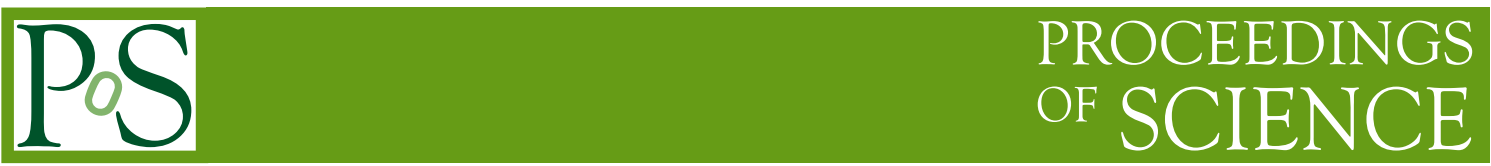

\title{
Measurement of the Differential Higgs Boson Production Cross Sections in the Leptonic WW Decay Mode with CMS
}

\author{
Sarah Freed on behalf of the CMS Collaboration ${ }^{a, *}$ \\ ${ }^{a}$ Rice University, \\ 6100 Main St., \\ Houston, Texas, USA \\ E-mail: sarah.m.freed@rice.edu
}

Measurements of differential production cross sections of the Higgs boson in pp collisions at $\sqrt{\mathrm{s}}=13 \mathrm{TeV}$ are performed using events where the Higgs boson decays into a pair of $\mathrm{W}$ bosons which subsequently decay into an electron, a muon, and a pair of neutrinos. The analysis is based on data collected by the CMS detector at the LHC in 2016, 2017, and 2018, and corresponds to an integrated luminosity of $137 \mathrm{fb}^{-1}$. Production cross sections with respect to the transverse momentum of the Higgs boson and the number of hadronic jets are considered. Higgs boson signal spectra are extracted and simultaneously unfolded to correct for selection efficiency and resolution effects by means of maximum likelihood fits to the observed event distributions. No significant deviation from the Standard Model expectations is observed in the differential measurements.

40th International Conference on High Energy physics - ICHEP2020

July 28 - August 6, 2020

Prague, Czech Republic (virtual meeting)

${ }^{*}$ Speaker 


\section{Introduction}

A study of the Higgs Boson decaying to two $\mathrm{W}$ Bosons with the different flavor leptonic $\left(\mathrm{e}^{ \pm} \mu^{\mp} v v\right)$ final state in pp collisions at $\sqrt{\mathrm{s}}=13 \mathrm{TeV}$ [1] was presented. The data used was collected by the CMS detector [2] in 2016, 2017 and 2018, corresponding to a luminosity of $137 \mathrm{fb}^{-1}$. The differential cross sections with respect to the transverse momentum of the Higgs Boson $\left(p_{\mathrm{T}}^{\mathrm{H}}\right)$ and the jet multiplicity $\left(N_{\text {jet }}\right)$ were measured. Measurements of the Higgs Boson properties in this decay mode are facilitated by the large branching fraction of the WW decay channel of the Higgs Boson, as well as the fact that the different flavor leptonic decay channel of the W Bosons is the cleanest. This makes it ideal for making precise measurements of the cross section, as well as making studies with the subleading Higgs Boson production modes. Additionally, the $\mathrm{e}^{ \pm} \mu^{\mp} v v$ final state is not required to be boosted, thus allowing use of the full $p_{\mathrm{T}}^{\mathrm{H}}$ range.

\section{Analysis Strategy}

The number of signal events is extracted using template fitting techniques. Since the dilepton mass $\left(m^{l l}\right)$ and the Higgs transverse mass $\left(m_{\mathrm{T}}^{\mathrm{H}}\right)$ were found to discriminate well against background, these are the variables used to extract the number of signal region events from the fit, which is done using $2 \mathrm{D}\left(m^{l l}, m_{\mathrm{T}}^{\mathrm{H}}\right)$ distributions. The signal region events are extracted in bins of $p_{\mathrm{T}}^{\mathrm{H}}$ and $N_{\text {jet }}$. Unfolding, regularization and signal extraction are all done simultaneously, with the exception of the $N_{\text {jet }}$ bins, where regularization is not needed. From this, we get the signal strength modifiers, $\mu_{i}$, which are taken to be equivalent to the scale factors from the fit in this analysis. From this, we get the cross section via $\mu_{i}=\sigma_{i}^{\text {obs }} / \sigma_{i}^{\mathrm{SM}}$.

The signal processes considered in this analysis are Higgs Boson production via gluon-gluon fusion (ggF), Vector Boson Fusion (VBF), weak-boson associated production (VH) and $t \bar{t}$ associated production $(\mathrm{tt} H)$ and are generated using POWHEG v2 at next-to-leading order (NLO) in QCD, with the ggF component reweighted to match NNLO accuracy. The dominant backgrounds in this analysis are Standard Model (SM) non-resonant $W W$ production $\left(W^{+} W^{-}\right)$, top pair production ( $\left.\overline{\mathrm{t}}\right)$, and Drell-Yan. Background processes are modeled mainly via POWHEG v2, MADGRAPH5_amc@nlo v2.4.2 and MCFM v7.0 at NLO in QCD. Drell-Yan and tt are constrained using control regions from data in the fit, but $W^{+} W^{-}$normalization is left free in the fit and is mainly constrained by the high $m^{l l}$ region. The non-dominant backgrounds consist of $W^{+} W^{-}$production via vector boson scattering, $\mathrm{V} \gamma / \mathrm{V} \gamma^{*}$, triboson production and non-prompt (fake) leptons. The analysis selections consist of two leptons with different flavor and opposite charge, leading (subleading) lepton $p_{\mathrm{T}}>25(13)$ $\mathrm{GeV},|\eta|<2.5, m^{l l}>12 \mathrm{GeV}$, dilepton transverse momentum $\left(p_{\mathrm{T}}^{l l}\right)>30 \mathrm{GeV}$, transverse mass of subleading lepton $\left(p_{\mathrm{T}}^{l_{2}}\right)>30 \mathrm{GeV}$ and $m_{\mathrm{T}}^{\mathrm{H}}>60 \mathrm{GeV}$. The signal region is further defined using missing transverse momentum $\left(p_{\mathrm{T}}^{\text {miss }}\right)>20 \mathrm{GeV}$ (final state neutrinos) and a b-jet veto to suppress top background. The top control region reverts the b-jet veto, requiring at least one b-tagged jet, and the Drell-Yan control region reverts the $m_{\mathrm{T}}^{\mathrm{H}}$ requirement.

\section{Results}

The observed differential cross sections, in bins of $N_{\text {jet }}$ and $p_{\mathrm{T}}^{\mathrm{H}}$, can be seen in Figure 1. There you can see the comparison of the observed cross section with the SM cross section. As 
previously mentioned, all samples in the nominal signal model are generated using POWHEG. The figures are also overlaid with the cross section prediction from an alternative model in which the MADGRAPH5_amc@NLO generator is used for ggF and VBF, while other components remain the same. The largest deviation from the SM can be found in the $N_{\text {jet }} \geq 4$ bin, and is 1.4 standard deviations. The main systematic uncertainties in this measurement come from the non-prompt lepton background, the $W^{+} W^{-}$background, residual $p_{\mathrm{T}}^{\text {miss }}$ uncertainties, and lepton ID efficiency scale factors.

Measurements of the signal strength modifier and cross section in the fiducial region were also done in this analysis and are reported here for the $p_{\mathrm{T}}^{\mathrm{H}}$ case, since it has smaller expected uncertainty. The fiducial region is defined using the same analysis selections which were defined in Analysis Strategy. Observed $\mu^{\text {fid }}$ was found to be:

$$
\mu^{\mathrm{fid}}=1.05 \pm 0.12( \pm 0.05(\text { stat }) \pm 0.07(\text { exp }) \pm 0.01(\text { signal }) \pm 0.07(\text { bkg }) \pm 0.03(\text { lumi }))
$$

Where (stat), (exp), (signal), (bkg) and (lumi) refer to statistical, experimental, signal (theoretical uncertainties related to modeling the signal), background, and luminosity uncertainties, respectively.

The observed cross section in the fiducial region $\left(\sigma^{\text {fid }}\right)$ is:

$$
\sigma^{\text {fid }}=86.5 \pm 9.5 \mathrm{fb}
$$

Which can be compared with the computed SM cross section, $\sigma^{\mathrm{SM}}=82.5 \pm 4.2 \mathrm{fb}$. Thus, we can see that the integrated fiducial cross section is consistent with the SM expectation.
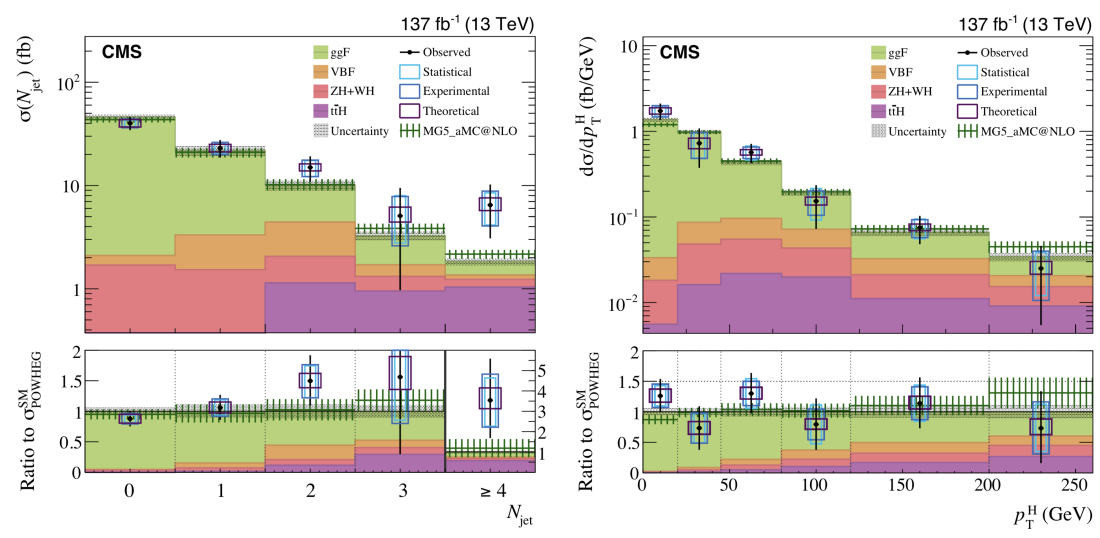

Figure 1: Observed differential fiducial cross sections, in bins of number of jets ( $N_{\text {jet }}$, on the left) and transverse momentum of the Higgs Boson ( $p_{\mathrm{T}}^{\mathrm{H}}$ on the right). Overlaid with predictions from nominal and alternative signal models. The filled histograms in the ratio plots show the relative contribution of the Higgs Boson production modes in each bin. [1]

\section{References}

[1] CMS Collaboration, "Measurements of differential Higgs boson production cross sections in the leptonic WW decay mode at $\sqrt{s}=13 \mathrm{TeV} "$, JHEP, arXiv:2007.01984

[2] CMS Collaboration, "The CMS experiment at the CERN LHC", JINST 3 (2008) S08004, doi:10.1088/1748-0221/3/08/S08004. 